\title{
Underwater Walking Intensity is Modified by a New and Untested Device that Increased the Lower Limb Surface of Movement
}

\author{
Marco Bergamin, ${ }^{1, *}$ Stefano Gobbo, ${ }^{1}$ Valentina Bullo, ${ }^{1}$ Federica Duregon, ${ }^{1}$ Barbara Vendramin, ${ }^{1}$ Cristine \\ Lima Alberton, ${ }^{2}$ Lucia Cugusi, ${ }^{3}$ Tobia Zanotto, ${ }^{4}$ Danilo Sales Bocalini, ${ }^{5}$ Andrea Di Blasio, ${ }^{6}$ Manuele
}

Bergamo, ${ }^{1}$ Marco Zaccaria, ${ }^{1}$ and Andrea Ermolao ${ }^{1}$

${ }^{1}$ Sport and Exercise Medicine Division, Department of Medicine, University of Padova, Italy

${ }^{2}$ Physical Education School, Federal University of Pelotas, Brazil

${ }^{3}$ Department of Medical Sciences, University of Cagliari, Italy

${ }^{4}$ Department of Clinical Exercise Physiology and Rehabilitation, Queen Margaret University of Edinburgh, United Kingdom

${ }^{5}$ Cardio-Physiology and Pathophysiology Laboratory, Federal University of Sao Paulo, Brazil

${ }^{6}$ Department of Medicine and Sciences of Aging, G. d'Annunzio University of Chieti-Pescara, Italy

"Corresponding author: Dr. Marco Bergamin, Sport and Exercise Medicine Division, Department of Medicine, University of Padova, Via Giustiniani, Padova, Italy. Tel: +39-0498214429, Fax: +39-0498215862, E-mail: marco.bergamin@unipd.it

Received 2017 May 23; Accepted 2017 August 07.

\begin{abstract}
Background: This study aimed to compare exercise intensity through measuring oxygen consumption $\left(\mathrm{VO}_{2}\right)$, heart rate $(\mathrm{HR})$, and rating of perceived effort (RPE) during an underwater walking at a standardized gait velocity $\left(\right.$ step $\left.\times \mathrm{min}^{-1}\right)$ when wearing swimsuit (SS) or aquatic pants (AP) that increased the drag force

Methods: 20 young healthy participants $(11 \mathrm{M}, 9 \mathrm{~F})$ were recruited; each participant was asked to perform two trials in a random order, one with SS, and the other with AP. Each trial consisted in a 20-minute walk on an underwater non-motorized treadmill at 60 , 70,80 , and 90 step $\times \min ^{-1}$.

Results: The total number of steps during the two trials and the walked distance was the same when wearing SS or AP at all rhythms. $\mathrm{VO}_{2}, \mathrm{HR}$, and RPE were significantly higher with the use of AP at all gait velocities $(\mathrm{P}<0.001)$.

Conclusions: Owing to the larger drag force, AP can be considered as an effective device to increase exercise intensity since the cardiorespiratory response was greater when wearing AP rather than SS.
\end{abstract}

Keywords: Aquatic Pants, Walking, Drag Force, Oxygen Consumption

\section{Background}

Exercising in water has become increasingly popular. These activities can be considered as safe and effective training methods to increase cardiorespiratory fitness (1), muscle strength (2), flexibility (3), and body composition (4). However, the main peculiarity of the water environment is represented by the dual-effect of buoyancy and resistance that requires higher levels of energy expenditure, with a relatively little movement or strain on lowjoint extremities (5). The water is an equalizing medium; its gravity-minimizing nature reduces compressive joint forces, providing a better exercise environment for people who do not tolerate weight-bearing activities $(6,7)$. These improvements were demonstrated in people at all ages $(8$, 9).

During the water-based exercise, the intensity can be increased with different methods. In primis, the simplest way is to increase the executive pace of a movement (10). In the use of devices (i.e. dumbbells) with different buoyancy coefficients, a higher intensity is produced in equal motion (11). Finally, another method to increase the exercise intensity can be obtained by maintaining the same movement pace and buoyancy coefficient, but modifying the dimension and the shape of a device. In that case, a higher drag force can be produced (12). The drag force is the mechanical force generated by the interaction between a solid body and a fluid (liquid or gas) acting oppositely to the relative motion of the body. The increase of the drag force is determined by the density and viscosity of the fluid, by the shape and the area of the body, and by the velocity of the body into the water. In particular, as velocity increases, drag force increases in a quadratic manner. Specifically, drag force can be calculated by the general fluid equation: $\mathrm{F}_{\mathrm{d}}=0.5 \times \mathrm{p} \times \mathrm{A} \times \mathrm{v}^{2} \times \mathrm{C}_{\mathrm{d}}$, where $\mathrm{p}$ is the density of water $\left(998.6 \mathrm{~kg} \times \mathrm{m}^{-3}\right)$, A is the projected frontal area (meters squared), $v^{2}$ is the velocity of the subject's body segments, and $C_{d}$ is the drag coefficient (13).

All of these features are important during the water- 
based exercise to regulate the intensity, adjust movement velocity and/or the surface of movement (14).

Although different studies have compared cardiorespiratory response to the exercise in water and land environments (15), only a few studies have analysed the same exercise performed on water with or without a device $(11,16)$.

Due to the increasing application of water-based activities and the lack of comparisons between physiological responses to the use or not use of different apparatus, there is a vital need to obtain a better understanding of the cardiorespiratory responses during exercises performed at different intensities with the use of dragging equipment. Therefore, the aim of this investigation was to observe the potential increase of the drag force wearing a new type of equipment. To verify this, we compared the exercise intensity during an underwater treadmill walking at four different gait velocities wearing a standard swimsuit (SS) or aquatic pants (AP) to increase underwater surface of displacement. We hypothesized that wearing the AP might increase oxygen consumption $\left(\mathrm{VO}_{2}\right)$, heart rate $(\mathrm{HR})$, and rating of perceived effort (RPE) during the same exercise without any equipment.

\section{Methods}

Twenty participants (11 M and $9 \mathrm{~F}$ ) were recruited among University students. The mean age was $24.35 \pm 2.8$ years, and the mean height and weight were $1.73 \pm 0.08$ $\mathrm{m}$ and $68.35 \pm 12.93 \mathrm{~kg}$, respectively, with averagely normoweight $\left(\mathrm{BMI}=22.70 \pm 3.53 \mathrm{~kg} \times \mathrm{m}^{-2}\right)$. The research protocol was performed under supervision of the sport and exercise Medicine division staff of the Department of Medicine at the University of Padova. Eligibility criteria included age between 18 and 35 years, body mass index (BMI) between 18.5 and $29.9 \mathrm{~kg} / \mathrm{m}^{2}$, and no other health problems and/or any physical limitations that could affect the study results. Information on the purpose and procedures of the study was given to each participant, and written consent was obtained before participation. The study complied with the current laws of Italy for research on human participants. The investigation procedure was examined and approved by the University of Padova review board (Ethics Board).

The test protocol was carried out in a swimming pool facility with a standardized environment; water temperature was set at approximately $29^{\circ} \mathrm{C}$ and temperature of air was between $26^{\circ} \mathrm{C}$ and $30^{\circ} \mathrm{C}$ (mean $27.28 \pm 1.65^{\circ} \mathrm{C}$ ) with $50 \%$ humidity, as recommended for a typical water fitness class (17). Before testing, the height and weight were measured respectively to the nearest $\mathrm{cm}$ using a stadiometer (Ayrton Corporation, Model S100, Prior Lake, MN) and to the nearest $0.1 \mathrm{~kg}$ using an electronic scale (home health care digital scale, model MC-660, C-7300 v1.1). Weight, height, age, and sex were recorded into a computer and a gas analyser. During each session, the participants were outfitted with a breathing mask for expiratory gas collection as well as a heart rate monitor, placed at the level of the xiphoid appendix. Two days before the trial session, the participants were instructed to consume standardized meals ( $55 \%$ carbohydrate, $30 \%$ fat, and $15 \%$ protein) and to abstain from caffeinated food or beverages 3-hour before the sessions. The protocol consisted of walking on an underwater non-motorized treadmill (Aqquatix, IT) with water at the waist level. The pool depth was $1.4 \mathrm{~m}$ and the treadmill height was $0.2 \mathrm{~m}$. Body immersion was set before every trial, based on the height of the participant, using 4 adjustable pedestals under the treadmill. The two trials were performed during the same day, one wearing SS and the other wearing AP, in a random order. Before each trial, the participants performed 5 minutes of warm-up and got familiar with the treadmill. After a further 5-minute rest to re-establish the lower heart rate, the participants were instructed to walk following the rhythms dictated by an electronic metronome at 60, 70, 80, and 90 step $\times \mathrm{min}^{-1}$, each for 5 minutes. The rhythms were derived from an adapted protocol previously adopted by Alberton et al. (10) to reduce the gait cadence because we hypothesized that wearing the AP might increase the exercise intensity $\left(\mathrm{VO}_{2}, \mathrm{HR}\right.$, and RPE); therefore, higher velocities could be excessively difficult to maintain. Furthermore, we added a minute to every stage to facilitate the steady state. No interruptions were provided between stages; there was a rest period of about 45 minutes between the two trials.

The SS consisted of a swim brief for men and two-piece swimsuit for women. These SS were characterized by small surface, close fitting.

AP consisted of long pants, closed with elastic bands at the ankles and at waist, with rubber lines inside the internal surface of the pants front- and back-side. These rubber lines were designed to contrast the textile rolling during the underwater movement, thereby keeping the surface of the pants as wide as possible (Figure 1). In other words, when carrying out the leg movement, pants started to swell in the opposite direction, increasing the total surface. The pulmonary gas exchange $\left(\mathrm{VO}_{2}, \mathrm{~mL} \times \mathrm{kg} \times{ }^{-1} \mathrm{~min}^{-1}\right.$; $\left.\mathrm{VCO}_{2}, \mathrm{~mL} \times \mathrm{kg} \times{ }^{-1} \mathrm{~min}^{-1}\right)$ and heart rate $\left(\right.$ beat $\left.\times \mathrm{min}^{-1}\right)$ were recorded through a portable gas analyzer $\left(\mathrm{K}_{4} \mathrm{~b}^{2}\right.$, Cosmed, IT) and a waterproof heart rate monitor (Polar, FI), respectively. The portable gas analyzer was calibrated before each test, using a known ambient-air and sample gas references, while the turbine flow meter was calibrated with a syringe of 3-liter known volume, following the instruction of the producer. Validity and reliability of the device have been described elsewhere (18). The portable gas ana- 
lyzer was placed inside a waterproof box, which was suspended nearby the participant by means of stainless-steel support to protect the device from splashing water, according to a method previously described in similar protocols $(19,20)$. All data were recorded during both sessions, with a telemetry data transmission system from the device to the computer. RPE was scored using the Borg 6 - 20 scale at 30 seconds before every change of gait velocity; each participant was previously given instructions on the use of the scale. During both trials, an underwater camera (GoPro, US) recorded the treadmill flow; subsequently, the number of laps of the treadmill tape was manually accounted to calculate the distances performed during all phases in both trials.

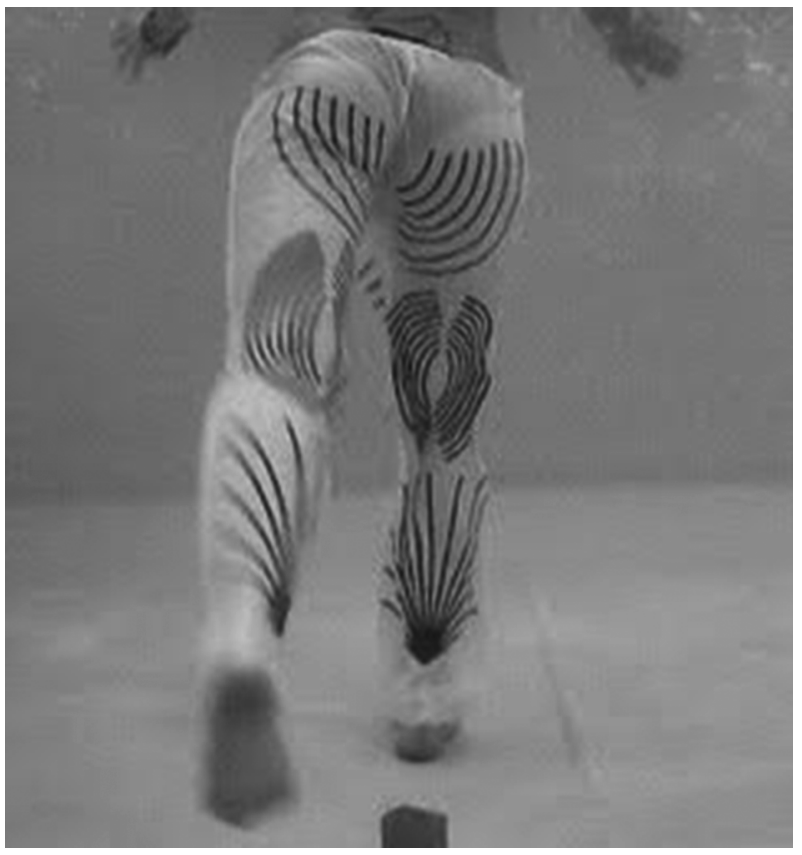

Figure 1. Aquatic Pants Closed with Elastic Bands at the Ankles and at Waist

Sample size calculation (N) was based on data reported in a similar protocol by Kanitz et al. (21). The sample size was calculated with the following equation for each condition: $\mathrm{N}=2 \mathrm{SD}^{2}\left(\mathrm{Z}_{\alpha}+\mathrm{Z}_{\beta}\right)^{2} / \Delta^{2}$. The equation estimated a sample size of 16.97 that would reflect a result with $80 \%$ power. To ensure an adequate sample size, we oversampled at 20 to allow for a $20 \%$ dropout rate due to technical problems or lack of participants interest.

Statistical analyses were carried out using SPSS (version 18.0 for Windows; IBM, Armonk, NY, USA). Results are expressed as means \pm standard deviation or percentage. The Shapiro-Wilk test demonstrated a normal distribution of the data. To examine the interaction between the four gait velocities $\left(60,70,80,90\right.$ steps $\left.\times \mathrm{min}^{-1}\right)$ and the two conditions (AP vs. SS) for all dependent variables, a $4 \times 2$ withinwithin model analysis of variance was applied. Fisher's least significant difference post-hoc contrasts with Bonferroni correction were applied to the different step rhythms. Linear regressions $\left(\mathrm{R}^{2}\right)$ were also calculated. Significance limits were set at the alpha level of $\mathrm{P}=0.05$.

\section{Results}

All participants completed both trials. No adverse effects or safety concerns were found during the whole exercise protocol. The mean of the last two minutes of each stage (from 3rd to 5 th minute) of $\mathrm{VO}_{2}$ and $\mathrm{HR}$ was reported as the mean \pm standard deviation and/or percentage (see Table 1).

First, taking in exam the gait velocity alone, analysis of variance indicated statistically significant differences for all the outcome variables: $\mathrm{VO}_{2}(\mathrm{~F}=105.027, \mathrm{P}<0.001)$, HR $(\mathrm{F}=98.435, \mathrm{P}<0.001), \mathrm{RPE}(\mathrm{F}=250.372, \mathrm{P}<0.001)$. Finally, there was a statistically significant difference in the distance performed at the different gait velocities $(F=288.030$, $\mathrm{P}<0.001)$.

Examining AP vs. SS, results indicated that when wearing AP, a statistically significant higher exercise intensity was determined $\left(\mathrm{VO}_{2}, \mathrm{~F}=34.428 ; \mathrm{P}<0.001 ; \mathrm{HR}, \mathrm{F}=33.839, \mathrm{P}\right.$ $<0.001$; and RPE, $\mathrm{F}=26.364, \mathrm{P}<0.001)$. On the other hand, the overall distance performed during both trials was the same, with no differences between the trials with or without the use of pants $(F=4.000, P=0.998)$; this means that during both trials, the participants developed the same path length.

Post-hoc contrasts showed averagely higher exercise intensity with AP, for all steps. Table 1 reports a higher $\mathrm{VO}_{2}$ value for AP than SS (15.73\% at 60 steps $\times \min ^{-1}, 12.02 \%$ at 70 steps $\times \min ^{-1}, 13.07 \%$ at 80 steps $\times \min ^{-1}$, and $8.03 \%$ at 90 steps $\times \mathrm{min}^{-1}$ ). The HR showed similar trend: $8.73 \%$, 8.45\%, $9.59 \%$, and $7.36 \%$ respectively at $60,70,80$, and 90 steps $\times$ $\mathrm{min}^{-1}$. Moreover, statistically significant higher RPE values were detected when wearing $\mathrm{AP}\left(10.14 \%\right.$ at 60 steps $\times \mathrm{min}^{-1}$, $8.56 \%$ at 70 steps $\times \min ^{-1}, 6.99 \%$ at 80 steps $\times \min ^{-1}$, and $10.16 \%$ at 90 steps $\times \min ^{-1}$ ). Levels of significance are reported in Table 1. Finally, complex-modelling ANOVA did not detect any interaction effect between the four performing rhythms and the use or not use of AP for all parameters $\left(\mathrm{VO}_{2}, \mathrm{~F}=0.940, \mathrm{P}=0.427 ; \mathrm{HR}, \mathrm{F}=0.759, \mathrm{P}=0.521\right.$; RPE, $\mathrm{F}=0.733, \mathrm{P}=0.073$; distance $, \mathrm{F}=0.503, \mathrm{P}=0.789$ ). Figure 2 shows relations between $\mathrm{VO}_{2}$ and $\mathrm{HR}$ for AP and SS conditions. Regression lines were $y=2.22814 \mathrm{x}+75.51\left(\mathrm{R}^{2}=0.3931\right.$, $\mathrm{P}<0.01)$ and $\mathrm{y}=2.2262 \mathrm{x}+71.35\left(\mathrm{R}^{2}=0.4081, \mathrm{P}<0.01\right)$ respectively for AP and SS. This appears to indicate an overall and harmoniously higher exercise intensity when walk- 
Table 1. Results ${ }^{\mathrm{a}}$

\begin{tabular}{|c|c|c|c|}
\hline \multicolumn{4}{|c|}{$\mathrm{VO}_{2}\left(\mathrm{~mL} \times \mathbf{k g}^{-1} \times \min ^{-1}\right)$} \\
\hline Steps & Swimsuit & $\mathbf{A P}$ & $\Delta^{\mathbf{b}} ;$ P value \\
\hline $1\left(60\right.$ steps $\left.\times \min ^{-1}\right)$ & $15.04 \pm 2.33$ & $17.41 \pm 3.57$ & $2.37 ; \mathrm{P}<0.001$ \\
\hline 2 (70 steps $\left.\times \mathrm{min}^{-1}\right)$ & $18.18 \pm 20.36$ & $20.36 \pm 4.26$ & $2.18 ; \mathrm{P}<0.001$ \\
\hline $3\left(80\right.$ steps $\left.\times \mathrm{min}^{-1}\right)$ & $21.08 \pm 4.53$ & $23.84 \pm 5.14$ & $2.76 ; \mathrm{P}<0.001$ \\
\hline $4\left(90\right.$ steps $\left.\times \min ^{-1}\right)$ & $25.39 \pm 6.37$ & $27.42 \pm 6.07$ & $2.03 ; \mathrm{P}<0.001$ \\
\hline \multicolumn{4}{|l|}{ HR $\left(\right.$ beat $\left.\times \min ^{-1}\right)$} \\
\hline Steps & Swimsuit & $\mathbf{A P}$ & $\Delta ;$ Pvalue \\
\hline $1\left(60\right.$ steps $\left.\times \min ^{-1}\right)$ & $99.2 \pm 12.44$ & $107.86 \pm 12.39$ & $8.66 ; \mathrm{P}<0.001$ \\
\hline $2\left(70\right.$ steps $\left.\times \min ^{-1}\right)$ & $110.91 \pm 13.99$ & $120.29 \pm 14.99$ & $9.38 ; \mathrm{P}<0.001$ \\
\hline $3\left(80\right.$ steps $\left.\times \min ^{-1}\right)$ & $121.02 \pm 17.35$ & $132.63 \pm 19.47$ & $11.61 ; \mathrm{P}<0.001$ \\
\hline 4 (90 steps $\left.\times \mathrm{min}^{-1}\right)$ & $134.47 \pm 20.81$ & $144.37 \pm 22.16$ & $9.9 ; \mathrm{P}<0.001$ \\
\hline \multicolumn{4}{|c|}{ RPE (Borg 6 - 20 scale) } \\
\hline Steps & Swimsuit & AP & $\Delta ;$ Pvalue \\
\hline 1 (60 steps $\left.\times \mathrm{min}^{-1}\right)$ & $6.9 \pm 1.29$ & $7.6 \pm 1.88$ & $0.7 ; \mathrm{P}<0.001$ \\
\hline 2 (70 steps $\left.\times \min ^{-1}\right)$ & $9.35 \pm 1.76$ & $10.15 \pm 1.95$ & $0.8 ; \mathrm{P}<0.001$ \\
\hline $3\left(80\right.$ steps $\left.\times \mathrm{min}^{-1}\right)$ & $11.45 \pm 1.43$ & $12.25 \pm 1.71$ & $0.8 ; \mathrm{P}<0.001$ \\
\hline $4\left(90\right.$ steps $\left.\times \min ^{-1}\right)$ & $12.8 \pm 1.11$ & $14.1 \pm 1.41$ & $1.3 ; \mathrm{P}<0.001$ \\
\hline
\end{tabular}

Abbreviations: AP, Aquatic Pants; RPE, Rating of Perceived Effort.

${ }^{\mathrm{a}} \mathrm{P}$ indicates the level of statistical significance.

${ }^{\mathrm{b}} \Delta$ (absolute change).

ing with AP; in fact, significant but weak correlations were found and regression equation trends were parallel with a higher value at the intersection in the y-axis for the AP regression line.

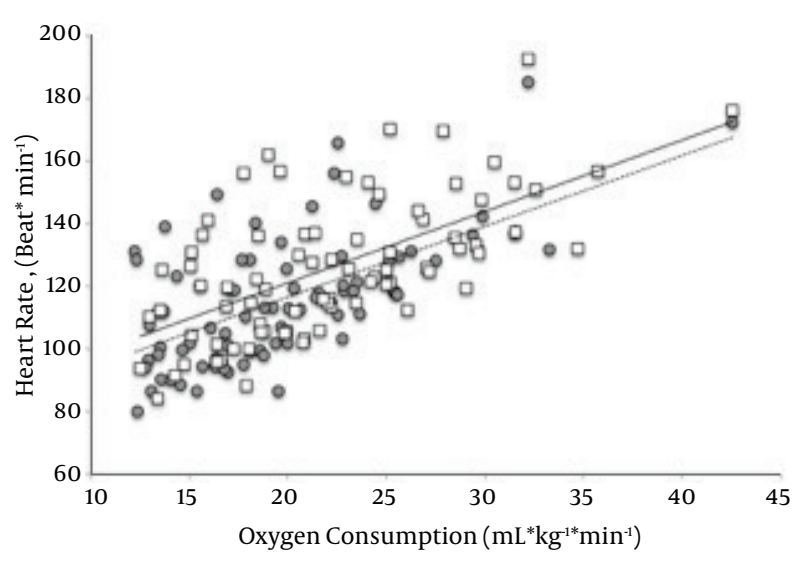

Figure 2. Relations Between $\mathrm{VO}_{2}$ and Heart Rate for Aquatic Pants and Swimsuit Conditions

\section{Discussion}

This investigation aimed to measure the potential increase in exercise intensity wearing a particular type of pants designed to develop the surface of movement during water-based exercise. For this purpose, cardiorespiratory parameters during underwater non-motorized treadmill walking were compared in two conditions, one wearing a traditional SS and the other with AP.

The main result of this study was that $\mathrm{HR}, \mathrm{VO}_{2}$, and RPE outcomes were significantly greater during all the four gait velocities when wearing AP rather than SS. Indirectly, it can be speculated that the conceivable increase of drag force could have determined an overall higher cardiorespiratory response. Apparently, at higher gait velocities, the difference between the two conditions (AP vs. SS) was lower. This flattening effect was more evident observing the tendency in the $\mathrm{VO}_{2}$ parameter, where there was the highest difference during the lowest gait speed (15.75\% of $\mathrm{VO}_{2}$ at 60 step $\left.\times \mathrm{min}^{-1}\right)$ and the smallest variation at the highest gait speed $\left(8.03 \%\right.$ of $\mathrm{VO}_{2}$ at 90 step $\left.\times \mathrm{min}^{-1}\right)$. Similarly, HR outcome also showed the same trend: $8.73 \%$ at 60 step $\times \min ^{-1}$ and $7.36 \%$ at 90 step $\times \min ^{-1}$. On the contrary, RPE values did not show any trend, with unexpected higher 
differences at 60 and 90 step $\times \min ^{-1}$ velocities. These nonlinear differences $\left(\mathrm{VO}_{2}\right.$ and $\left.\mathrm{HR}\right)$ could be ascribed to the variation of the AP surface during the different velocities. It is likely that at higher velocities, the AP modified its shape tending to flatten onto the limb and then reducing the total surface of movement; this effect could have determined a variation in the drag coefficient.

Literature reported a variable relationship between $\mathrm{HR}$ and $\mathrm{VO}_{2}$, depending on the type of aquatic exercise (22), intensity (23), water depth (19), and temperature (20). Similar to Shono et al. investigations $(24,25)$, our analysis revealed a linear relationship between $\mathrm{HR}$ and $\mathrm{VO}_{2}$ in both conditions. Despite coefficients of determination that were moderate for the AP and the SS $\left(\mathrm{R}^{2} \approx 0.4\right)$, regression lines were comparable with equivalent slopes (2.28 for AP and 2.26 for SS, Figure 2). Interestingly, the AP $\mathrm{HR}-\mathrm{VO}_{2}$ ratio was higher compared to that of SS indicating that similar $\mathrm{VO}_{2}$ levels corresponded a larger $\mathrm{HR}$ response in AP condition. This could support the hypothesis that during AP condition, a higher cardiorespiratory intensity is needed to perform the same walking exercise at the same gait velocity.

From a practical perspective, the drag force can be easily modified for instance by walking against an artificial current (26) or adding a water-jet (27); however, these conditions are easily reproducible in a laboratory setting, but with a concrete difficult application in the field. In contrast to the larger amount of data about devices to increase the buoyancy (28) that are also largely commercially available, only a few investigations described wearable devices developed to increase the drag force. De Souza et al. (16) described positive results using a water-dragging device. Concretely, they compared the energy expenditure during a session of strength training performed with and without that device showing a post-exercise higher resting metabolic rate. In another study, Pinto and Colleagues (1) compared $\mathrm{HR}, \mathrm{VO}_{2}$ and electromyographic signals during exercise with a water-drag force equipment, water-floating equipment, and without any equipment. The $\mathrm{HR}$ and $\mathrm{VO}_{2}$ showed significantly higher values during the aquatic workout with both devices compared to the same performance without equipment. More interestingly, a greater electromyographic response was seen using the drag force device than with the water-floating equipment, which in turn suggested a greater resistance to the movement in that condition.

This investigation presents several limitations. The main limit is probably due to that the AP has three sizes. We selected the appropriate size following the waist circumference as a standard criterion. This entailed that a non-uniform drag force was probably produced. Another critical point consisted in the positioning of the under- water non-motorized treadmill. The treadmill was placed near the poolside; then, waves generated by the lower limbs might have affected the surface movement of the AP. Finally, temperature and humidity variations could have slightly affected the reading of the respiratory volumes by the gas analyzer. Nonetheless, these variations should be considered as minimal and acceptable variations since punctual calibration of the gas analyzer was performed before all trials.

To summarize, the AP seemed to be an effective tool to increase exercise intensity during underwater walking. It was concretely difficult to make strict comparisons among our results and the other aforementioned results mainly due to that devices were basically different; second the types of exercise were not standardized (as the our) and finally some investigations focused on the elderly or other populations which were not similar to our sample. Furthermore, the effectiveness of AP to increase the intensity in other types of exercise rather than underwater walking has not been proven. Future protocols should take into consideration investigating other types of population, especially people who need to exercise with low movement velocity, in particular for knee and / or hip osteoarthritis, where aquatic protocols are strongly recommended (29). Another interesting point concerns the capacity of the underwater waves to create unbalanced conditions (4). The use of AP could further stress this phenomenon due to the larger surface; this may produce an increase in static and dynamic balance capacities. One non-secondary aspect of the use of AP concerns the possibility to hidden the own body from the other views during workout sessions. This should be taken into account when discussing about motivation to exercise (30) and explaining reasons for early dropouts, since the AP could help hide their own body, especially for those people who do not feel comfortable with their own body image.

In conclusion, the AP could be considered as an effective device to increase exercise intensity. The results of the present study indicated that AP was able to increase $\mathrm{VO}_{2}$, $\mathrm{HR}$, and RPE during an underwater walking in comparison with traditional swimsuit. The increase of drag force could have determined an overall higher cardiorespiratory response during the underwater walking. Nevertheless, the difference of cardio-metabolic parameters when wearing swimsuit or AP was not the same in each gait velocity. Finally, AP may be an effective device to increase exercise intensity and improve cardio-metabolic and physical fitness.

\section{Acknowledgments}

The authors declare no conflicts of interest and no sources of funding. This investigation was not financed by 
any institution or private entity.

\section{References}

1. Pinto SS, Cadore EL, Alberton CL, Silva EM, Kanitz AC, Tartaruga MP, et al. Cardiorespiratory and neuromuscular responses during water aerobics exercise performed with and without equipment. Int J Sports Med. 2011;32(12):916-23. doi: 10.1055/s-0031-1283176. [PubMed: 22052026].

2. Pinto SS, Alberton CL, Bagatini NC, Zaffari P, Cadore EL, Radaelli R, et al. Neuromuscular adaptations to water-based concurrent training in postmenopausal women: effects of intrasession exercise sequence. Age (Dordr). 2015;37(1):9751. doi: 10.1007/s11357-015-9751-7. [PubMed: 25643897].

3. Pinto SS, Cadore EL, Alberton CL, Zaffari P, Bagatini NC, Baroni BM, et al. Effects of intra-session exercise sequence during water-based concurrent training. Int J Sports Med. 2014;35(1):41-8. doi: 10.1055/s-00331345129. [PubMed: 23771835].

4. Bergamin M, Ermolao A, Tolomio S, Berton L, Sergi G, Zaccaria M. Water-versus land-based exercise in elderly subjects: effects on physical performance and body composition. Clin Interv Aging. 2013;8:110917. doi: $10.2147 / C I A . S 44198$. [PubMed: 24009416].

5. Alberton CL, Pinto SS, Cadore EL, Tartaruga MP, Kanitz AC, Antunes AH et al. Oxygen uptake, muscle activity and ground reaction force during water aerobic exercises. Int J Sports Med. 2014;35(14):1161-9. doi: 10.1055/s-0034-1383597. [PubMed: 25144436].

6. Meredith-Jones $\mathrm{K}$, Waters $\mathrm{D}$, Legge $\mathrm{M}$, Jones $\mathrm{L}$. Upright waterbased exercise to improve cardiovascular and metabolic health: a qualitative review. Complement Ther Med. 2011;19(2):93-103. doi: 10.1016/j.ctim.2011.02.002. [PubMed: 21549260].

7. Cugusi L, Cadeddu C, Nocco S, Orru F, Bandino S, Deidda M, et al. Effects of an aquatic-based exercise program to improve cardiometabolic profile, quality of life, and physical activity levels in men with type 2 diabetes mellitus. PM R. 2015;7(2):141-8. doi: 10.1016/j.pmrj.2014.09.004. [PubMed: 25217820] quiz 148.

8. Barbosa TM, Marinho DA, Reis VM, Silva AJ, Bragada JA. Physiological assessment of head-out aquatic exercises in healthy subjects: a qualitative review. J Sports Sci Med. 2009;8(2):179-89. [PubMed: 24149524].

9. Bergamin M, Zanuso S, Alvar BA, Ermolao A, Zaccaria M. Is water-based exercise training sufficient to improve physical fitness in the elderly? Eur Rev Aging Phys Act. 2012;9(2):129-41. doi:10.1007/s11556-012-0097-1.

10. Alberton CL, Cadore EL, Pinto SS, Tartaruga MP, da Silva EM, Kruel LF. Cardiorespiratory, neuromuscular and kinematic responses to stationary running performed in water and on dry land. Eur J Appl Physiol. 2011;111(6):1157-66. doi: 10.1007/s00421-010-1747-5. [PubMed: 21127897].

11. Colado JC, Borreani S, Pinto SS, Tella V, Martin F, Flandez J, et al. Neuromuscular responses during aquatic resistance exercise with different devices and depths. J Strength Cond Res. 2013;27(12):3384-90. doi: 10.1519/JSC.ob013e3182915ebe. [PubMed: 23539078].

12. Colado JC, Garcia-Masso X, Gonzalez LM, Triplett NT, Mayo C, Merce J. Two-leg squat jumps in water: an effective alternative to dry land jumps. Int J Sports Med. 2010;31(2):118-22. doi: 10.1055/s-0029-1242814. [PubMed: 20222004].

13. Poyhonen T, Keskinen KL, Hautala A, Malkia E. Determination of hydrodynamic drag forces and drag coefficients on human leg/foot model during knee exercise. Clin Biomech (Bristol, Avon). 2000;15(4):256-60. doi: 10.1016/S0268-0033(99)00070-4. [PubMed: 10675666].

14. Colado JC, Triplett NT. Monitoring the intensity of aquatic resistance exercises with devices that increase the drag force: An update. Strength Cond J. 2009;31(3):94-100. doi:10.1519/SSC.0b013e3181a605b2.

15. Heywood S, McClelland J, Geigle P, Rahmann A, Clark R. Spatiotemporal, kinematic, force and muscle activation outcomes during gait and functional exercise in water compared to on land: A systematic review. Gait Posture. 2016;48:120-30. doi: 10.1016/j.gaitpost.2016.04.033. [PubMed: 27236637].

16. de Souza AS, Pinto SS, Kanitz AC, Rodrigues BM, Alberton CL, da Silva EM, et al. Physiological comparisons between aquatic resistance training protocols with and without equipment. J Strength Cond Res. 2012;26(1):276-83. doi: 10.1097/ICO.0b013e31821e429d. [PubMed: 22193342].

17. Barbosa TM, Garrido MF, Bragada J. Physiological adaptations to head-out aquatic exercises with different levels of body immersion. J Strength Cond Res. 2007;21(4):1255-9. doi:10.1519/R-20896.1. [PubMed: 18076241].

18. Duffield R, Dawson B, Pinnington HC, Wong P. Accuracy and reliability of a Cosmed K4b2 portable gas analysis system. J Sci Med Sport. 2004;7(1):11-22. doi: 10.1016/S1440-2440(04)80039-2. [PubMed: 15139160].

19. Benelli P, Colasanti F, Ditroilo M, Cuesta-Vargas A, Gatta G, Giacomini $F$, et al. Physiological and biomechanical responses to walking underwater on a non-motorised treadmill: effects of different exercise intensities and depths in middle-aged healthy women. J Sports Sci. 2014;32(3):268-77. doi: 10.1080/02640414.2013.824601. [PubMed 24015764].

20. Bergamin M, Ermolao A, Matten S, Sieverdes JC, Zaccaria M. Metabolic and cardiovascular responses during aquatic exercise in water at different temperatures in older adults. Res Q Exerc Sport. 2015;86(2):16371. doi: 10.1080/02701367.2014.981629. [PubMed: 25513937].

21. Kanitz AC, Liedtke GV, Pinto SS, Alberton CL, Kruel LFM. Cardiorespiratory responses during deep water running with and without horizontal displacement at different cadences. Rev Andal Med Deporte. 2014;7(4):149-54. doi:10.1016/j.ramd.2014.07.002.

22. Ruoti RG, Troup JT, Berger RA. The effects of nonswimming water exercises on older adults. J Orthop Sports Phys Ther. 1994;19(3):140-5. doi 10.2519/jospt.1994.19.3.140. [PubMed: 8156064].

23. Sheldahl LM, Tristani FE, Clifford PS, Hughes CV, Sobocinski KA, Morris RD. Effect of head-out water immersion on cardiorespiratory response to dynamic exercise. J Am Coll Cardiol. 1987;10(6):1254-8. doi 10.1016/S0735-1097(87)80127-4. [PubMed: 3680793].

24. Shono T, Fujishima K, Hotta N, Ogaki T, Ueda T. Physiological responses to water-walking in middle aged women. J Physiol Anthropo Appl Human Sci. 2001;20(2):119-23. doi: 10.2114/jpa.20.119. [PubMed: 11385934].

25. Shono T, Fujishima K, Hotta N, Ogaki T, Ueda T, Otoki K, et al. Physiological responses and RPE during underwater treadmill walking in women of middle and advanced age. J Physiol Anthropol Appl Human Sci. 2000;19(4):195-200. doi: 10.2114/jpa.19.195. [PubMed: 11037694].

26. Masumoto K, Hamada A, Tomonaga HO, Kodama K, Hotta N. Physiological responses, rating of perceived exertion, and stride characteristics during walking on dry land and walking in water, both with and without a water current. J Sport Rehabil. 2012;21(2):175-81. doi: 10.1123/jsr.21.2.175. [PubMed: 22104030].

27. Bressel E, Smith G, Miller A, Dolny D. Aquatic-treadmill walking: quantifying drag force and energy expenditure. J Sport Rehabil. 2012;Tech nical Notes(5) [PubMed: 22715134].

28. Costa G, Afonso S, Bragada JA, Reis VM, Barbosa TM. Comparison of acute physiological adaptations between three variants of a basic head-out water exercise. Rev Bras Cineantropom Desempenho Hum. 2008;10(4):323-9.

29. Kamioka H, Tsutani K, Mutoh Y, Okuizum H, Ohta M, Handa S, et al. A systematic review of nonrandomized controlled trials on the $\mathrm{cu}$ rative effects of aquatic exercise. Int J Gen Med. 2011;4:239-60. doi: 10.2147/IJGM.S17384. [PubMed: 21556311].

30. Lepore M, Gayle GW, Stevens SF. Adapted Aquatics Programming: A Professional Guide. Champain; 1998. 